\title{
Force-Sensitive Mat for Vertical Jump Measurement to Assess Lower Limb Strength: Validity and Reliability Study
}

Erik Vanegas $^{1 *}, \mathrm{MSc}$;olocuauhtli Salazar ${ }^{2 *}, \mathrm{PhD} ;$ Raúl Igual $^{1^{*}}, \mathrm{PhD} ;$ Inmaculada Plaza ${ }^{1^{*}}, \mathrm{PhD}$

${ }^{1}$ Electrical/Electronics Engineering and Communications Department, EUP Teruel, Universidad de Zaragoza, Teruel, Spain

${ }^{2}$ Tecnológico Nacional de México, IT Durango, Durango, Mexico

*all authors contributed equally

\section{Corresponding Author:}

Erik Vanegas, MSc

Electrical/Electronics Engineering and Communications Department, EUP Teruel

Universidad de Zaragoza

Atarazana 2

Teruel, 44003

Spain

Phone: 34978618102

Email: erikvanegas599@gmail.com

\section{Abstract}

Background: Vertical jump height is widely used in health care and sports fields to assess muscle strength and power from lower limb muscle groups. Different approaches have been proposed for vertical jump height measurement. Some commonly used approaches need no sensor at all; however, these methods tend to overestimate the height reached by the subjects. There are also novel systems using different kind of sensors like force-sensitive resistors, capacitive sensors, and inertial measurement units, among others, to achieve more accurate measurements.

Objective: The objective of this study is twofold. The first objective is to validate the functioning of a developed low-cost system able to measure vertical jump height. The second objective is to assess the effects on obtained measurements when the sampling frequency of the system is modified.

Methods: The system developed in this study consists of a matrix of force-sensitive resistor sensors embedded in a mat with electronics that allow a full scan of the mat. This mat detects pressure exerted on it. The system calculates the jump height by using the flight-time formula, and the result is sent through Bluetooth to any mobile device or PC. Two different experiments were performed. In the first experiment, a total of 38 volunteers participated with the objective of validating the performance of the system against a high-speed camera used as reference ( $120 \mathrm{fps})$. In the second experiment, a total of 15 volunteers participated. Raw data were obtained in order to assess the effects of different sampling frequencies on the performance of the system with the same reference device. Different sampling frequencies were obtained by performing offline downsampling of the raw data. In both experiments, countermovement jump and countermovement jump with arm swing techniques were performed.

Results: In the first experiment an overall mean relative error (MRE) of $1.98 \%$ and a mean absolute error of $0.38 \mathrm{~cm}$ were obtained. Bland-Altman and correlation analyses were performed, obtaining a coefficient of determination equal to $R^{2}=.996$. In the second experiment, sampling frequencies of $200 \mathrm{~Hz}, 100 \mathrm{~Hz}$, and $66.6 \mathrm{~Hz}$ show similar performance with MRE below $3 \%$. Slower sampling frequencies show an exponential increase in MRE. On both experiments, when dividing jump trials in different heights reached, a decrease in MRE with higher height trials suggests that the precision of the proposed system increases as height reached increases.

Conclusions: In the first experiment, we concluded that results between the proposed system and the reference are systematically the same. In the second experiment, the relevance of a sufficiently high sampling frequency is emphasized, especially for jump trials whose height is below $10 \mathrm{~cm}$. For trials with heights above $30 \mathrm{~cm}$, MRE decreases in general for all sampling frequencies, suggesting that at higher heights reached, the impact of high sampling frequencies is lesser.

(JMIR Mhealth Uhealth 2021;9(4):e27336) doi: $10.2196 / 27336$

\section{KEYWORDS}

vertical jump; mHealth; mobile health; force-sensitive resistor; lower limb strength; leg strength 


\section{Introduction}

Vertical jump height is one of the physical skills commonly used to assess overall performance in human beings, and more specifically, it is used to assess performance and muscle power of the quadriceps, hamstrings, and gastrocnemius muscle groups in the lower limbs [1,2]. Measurement of the performance of this skill is commonly performed on athletes in sports like basketball [3,4], football [5], netball [6], swimming [7], and others. This skill performance can also provide important data from people with no relevant sports past.

In the literature, there are many protocols to prove or validate the proposed systems. Among the different kind of jumps performed in those protocols, there are jumps with and without countermovement [1,4,5,8-15], jumps with and without arm swing $[12,16]$, drop jumps $[1,8,17]$, single and double leg jump [6], continuous jumps [4,17], squat jumps [1,2,4,12], and loaded squat jumps [7]. With any of these types of jumps, height reached by the user can be analyzed, but the jumps most commonly used in all related work are the countermovement and squat jumps.

Kibele [15] and Moir [18] reported that irregularities detected in measurements of any kind of jump execution may be linked to changes in the posture of a subject during flight due to change in the center of mass of the subject during the jump. Bui et al [13] found that some common errors obtained during measurement were caused by body movements like knee, hip, and ankle bending during flight time and landing. Also, Aragon-Vargas [19] states that ascending and descending phases of flight time must be of the same length of time, but in his work descending time was significantly longer, suggesting that participants descended with their bodies partially crouched.

There are several techniques to measure jump height, each of which uses a different kind of sensor or no sensors at all. Methods like the Sargent jump [13] and Vertec device [6,9,10] require no sensors and often are used as reference measurements. However, these methods often show overestimation on jump height, and this could be due to arm stretching performed unconsciously by the user. Among systems developed in the literature, different kind of sensors are used like force-sensitive resistors (FSRs) [3,16], capacitive sensors [5], inertial measurement units $[2,4,8,10,17]$, electromyography sensors [1,6], kinematic sensors [6], ultrasonic sensors [20], microswitches [9], video cameras, [11] and optical sensors $[4,12]$.

The studies of Drazan et al [3] and Boukhenous and Attari [16] are most closely related to our work, as both of their systems also use resistive sensors. However, only one sensor is used for the whole sensing area. Drazan et al [3] proposed a system based on a single FSR sensor whose total sensing area is around 3 $\mathrm{cm}^{2}$, with an Arduino board as microcontroller. This system calculated jump height through the flight-time formula, by measuring the time the FSR sensor is not detecting any pressure. In the work presented by Boukhenous and Attari [16], two metallic strain gauges were placed in the center of a rigid platform to measure the force applied by the ground. In this case, vertical ground reaction forces were used to calculate jump height. Rico et al [2] used pressure sensors located at the forefoot of the user to calculate flight time during vertical jump and compare it with data obtained from an inertial measurement unit system. However, few or no specific number of subjects are used in these studies, and no specific protocols or jump trials are performed. Also, no reference systems are used to compare the performance of the developed systems.

Camera-based systems are used as reference systems or as proposed methods to validate. In some studies, the famous motion capture system commonly used in videogames is used as a reference device $[5,17,21]$. Other studies use a similar method by tracking body markers placed strategically on the body [4,14,21]. Balsalobre-Fernandez et al [11,22] have analyzed the effectiveness and reliability of high-speed cameras as methods to estimate vertical jump height. In these studies, flight time of the subject is calculated by selecting the takeoff and landing frames of the recorded videos of jump trials, and, by applying the flight-time formula, jump height is obtained $[3,8,9,11,14,16,22]$.

Only a few studies perform a validation with a relatively high number of subjects. Some studies that fulfill this criterion are the ones presented by Nuzzo et al [10], Casartelli et al [4], Glatthorn et al [12], Moir [18], and Aragon-Vargas [19]. However, these studies compare different commercially available devices (contact mat, force plate, cameras, etc), and no novel system is developed by the researchers. Bui et al [13] fulfills the criterion and proposes a novel optical system whose performance is compared against commercial devices. This system calculates jump height through the flight-time formula.

This study presents a newly developed low-cost system for measuring height reached by users during vertical jump comprising a matrix of FSR sensors embedded on a mat. The height of the vertical jump is calculated through the flight-time formula $[3,8,9,14,16]$. One advantage this system offers against other pressure-sensitive systems $[2,3,16]$ is a higher real sensing area, higher resolution, and higher precision, as this system works with 256 FSR sensors distributed around the mat in 16 rows and 16 columns, in comparison with the other systems that use a single sensor. The total sensing area, dimensions of FSR sensors matrix, and each individual FSR sensor area can be modified on different versions of the proposed mat. Also, as this system is environmental, it needs no adjustment regardless of the physical characteristics of the user like body type, weight, height, or foot size [2]. Another advantage of this system is that the calculated vertical jump height is directly sent to a PC or mobile device of the health care professional's choice, unlike other methods that require postprocessing analysis, as in the high-speed camera method. The main objective of this study is to validate the reliability of the proposed system for future clinical studies.

\section{Methods}

\section{System Construction}

The proposed system consists of 2 parts: a resistive pressure-sensitive mat constructed with an FSR sensor array and the electronic system. The mat is composed of 3 layers. 
One layer contains thin and flat copper wires distributed in a column arrangement along a flexible 3D printed grid; another layer is composed of the same copper wires but distributed in a row arrangement on another flexible 3D printed grid. A third layer is placed between the layers consisting of Velostat material, a pressure-sensitive material that behaves as a resistor whose value drops whenever pressure is exerted upon it. In this way, variations on the resistance values on every intersection of rows and columns when pressure is exerted over the mat can be measured. Some typical characteristic problems with Velostat material are repeatability, nonlinearity, and hysteresis [23,24]. For this application, however, these features are not relevant because high precision is not needed; the mat only needs to be able to detect a heavy body placed on it (average human body weight). More information about the development of this mat is documented on the work of Medrano et al [25].

Figure 1 shows the different layers of an FSR matrix with smaller dimensions (4 rows and 4 columns). Figure 2 shows an example of the placement of the overlapped layers. The total sensing area of the mat used for this study is $30 \times 30 \mathrm{~cm}$, with 16 rows and 16 columns, $1 \mathrm{~cm}$ width each. This way, the area of each of the individual FSR sensors is equal to $1 \mathrm{~cm}^{2}$. In Figure 3 , a developed mat is shown.

Figure 1. Different layers comprising a force-sensitive resistor matrix with smaller dimensions.

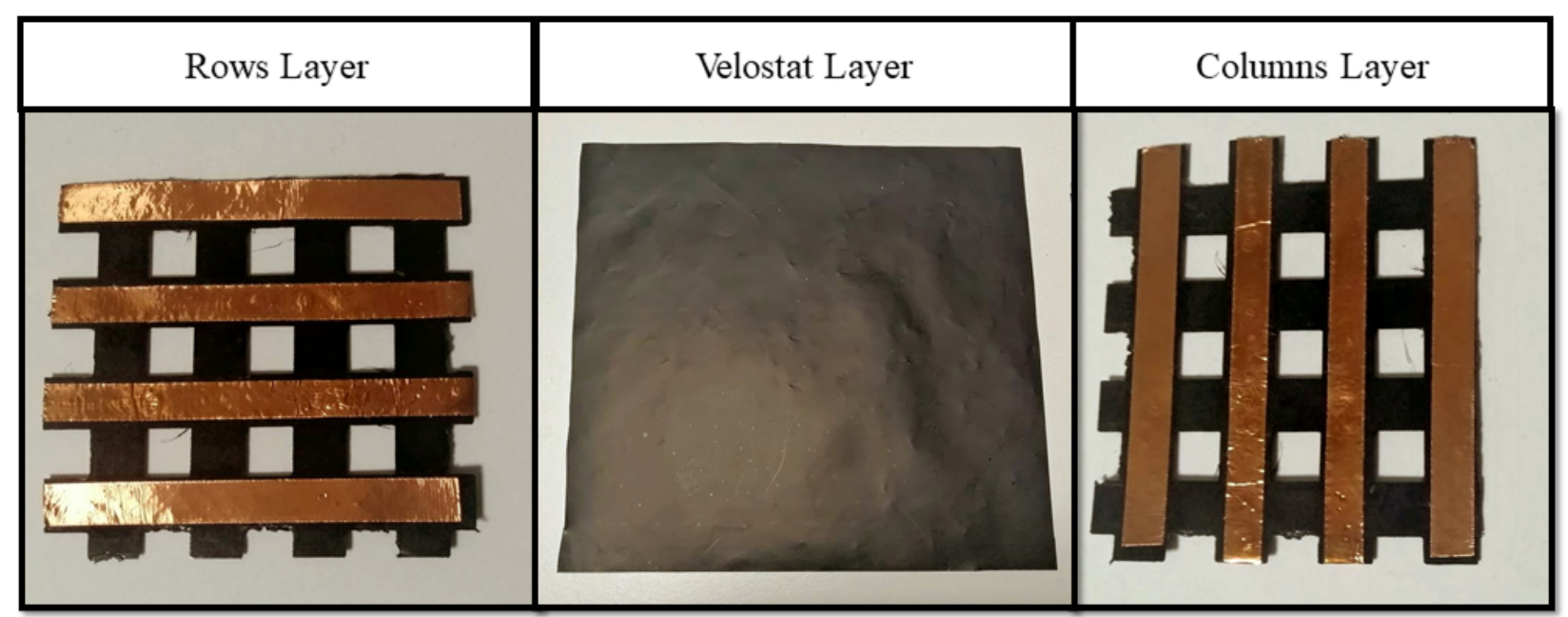

Figure 2. Example of a smaller size matrix and how layers are placed.

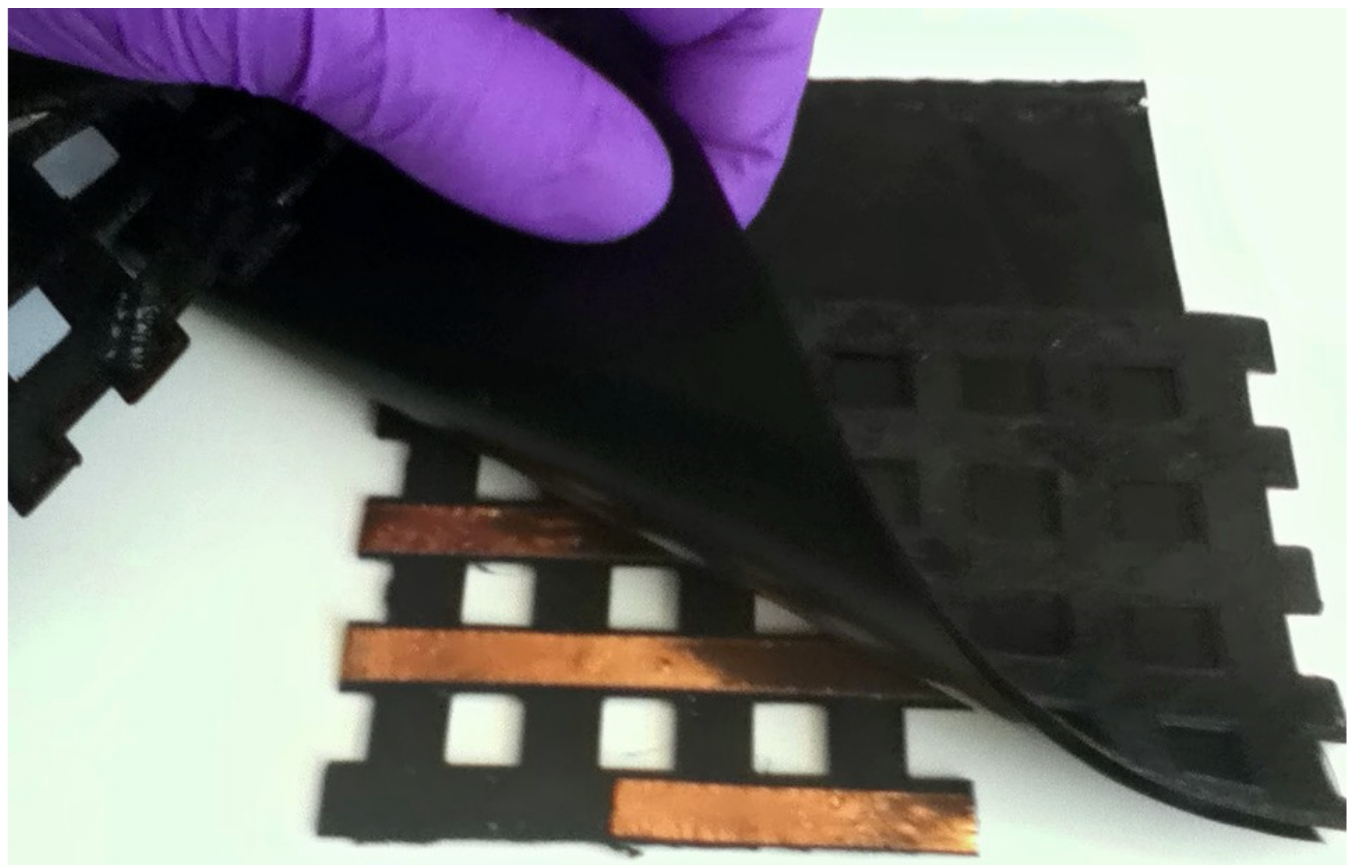


Figure 3. Developed force-sensitive resistor sensor mat.

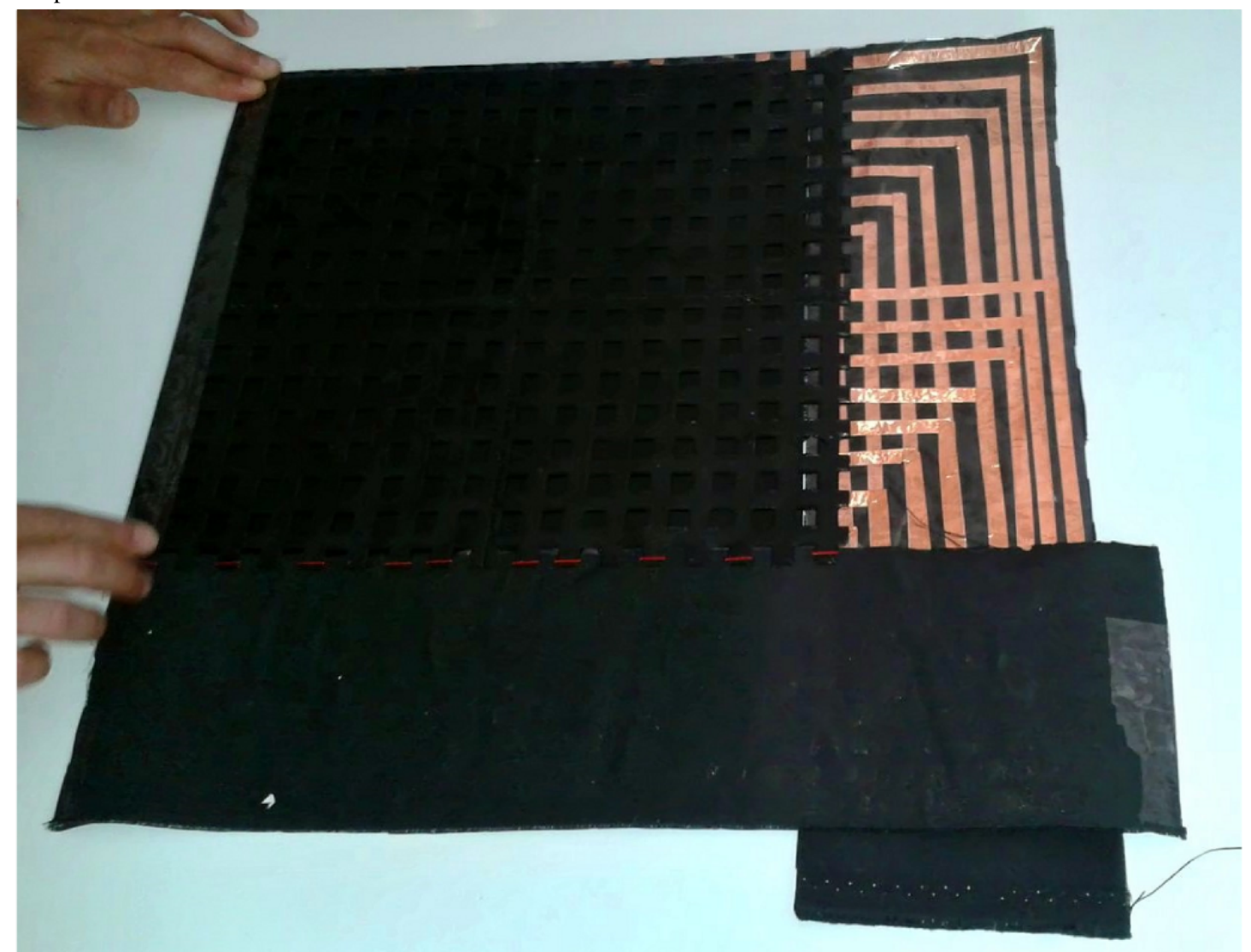

Due to the number of operations needed for a full scan of the mat, a high frequency microprocessor must be used for data processing, as the time complexity of these operations grows in exponential order. The STM32F103C8T6 microprocessor (STMicroelectronics) was selected due to its $72 \mathrm{MHz}$ CPU frequency, with which a sampling frequency of $200 \mathrm{~Hz}$ is achieved. Other microprocessors with lower CPU frequencies (like the ATmega328P, Microchip Technology Inc) would not achieve the desired sampling frequency. Also two 16-1 multiplexers 74HC4067 are needed for an efficient scan process of the whole mat. For data transmission, a Bluetooth HC-05 module is used. Bluetooth technology was selected due to its ease of connection with different devices, especially with smartphones and tablets, which offers health care professionals the choice of an easy-to-transport monitoring device. Other electronic elements included in the system are a TP4056 battery-charging module and a Lipo battery of $3.7 \mathrm{~V}$ and 150 $\mathrm{mAh}$ capacity, allowing continuous functioning of the system for up to $2 \mathrm{hrs}$. A block diagram of the proposed system is shown in Figure 4.

The algorithm used for this system consists of calculating the summation of every FSR sensor of the mat. For each FSR sensor, the voltage value obtained by the analog-to-digital converter of the microcontroller is given in bits (from 0 to 4095), and this resulting value is used for the calculations. A threshold is used for the system to decide whether a person is standing on the mat or not. To calculate an appropriate value for this threshold, data were collected from 16 volunteers ( 5 female and
11 male), with an average weight of 74.81 (SD 15.25) $\mathrm{kg}$ and foot size of 26.93 (SD 1.94) $\mathrm{cm}$. The volunteers were asked to stand on the mat barefoot in 4 different positions: with both feet standing still and on their forefoot and with one foot standing still and on their forefoot. Maximum values of pressure exerted on FSR sensors were used as reference for normalization, and the minimum value for activation of FSR sensors was considered as no volunteer standing on the mat.

Using such criteria, on average when standing still over the mat with both feet subjects activated $71.66 \%$ of the FSR sensors, and when standing on their forefoot with both feet, $28.9 \%$ of the FSR sensors were activated. When standing still and on their forefoot with only one foot an activation of $40.37 \%$ and $18.40 \%$ of the FSR sensors was registered, respectively. The minimum value of FSR sensors activation is registered when standing on one foot on their forefoot, with a value of $12.09 \%$. All results are summarized in Table 1. By taking these results into account, and if it is assumed that volunteers may land first with one foot on their forefoot after a jump, a proper threshold should be proposed below the minimum value of FSR sensors activation. For this study, a threshold of $9 \%$ of FSR sensors activation is used. This threshold was chosen to be at three-quarters between the zero FSR sensor activation and the minimum FSR sensor activation recorded, to avoid any misreading from mechanical oscillation. It is worth noting that the minimum recorded value from sensor activation is an outlier. In future studies, the possibility of adding a personalized threshold for every subject could be assessed. 
Figure 4. Block diagram of the proposed system.

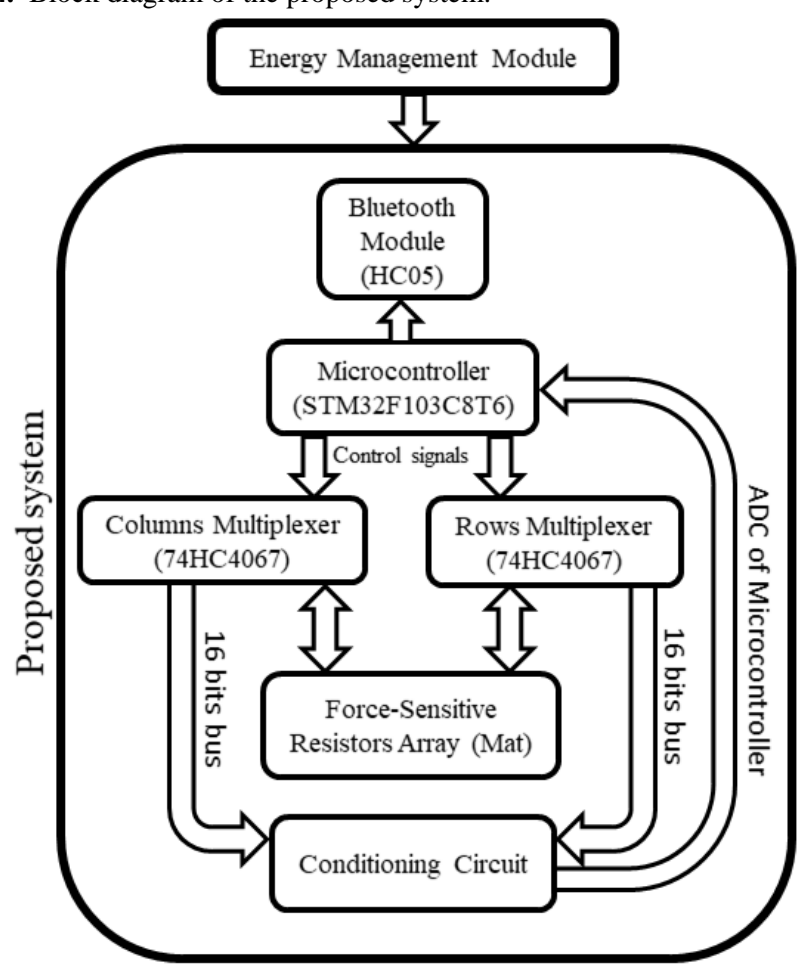

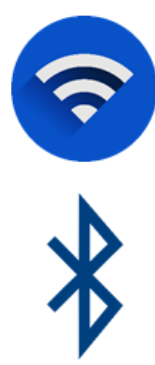

Bluetooth Transmission

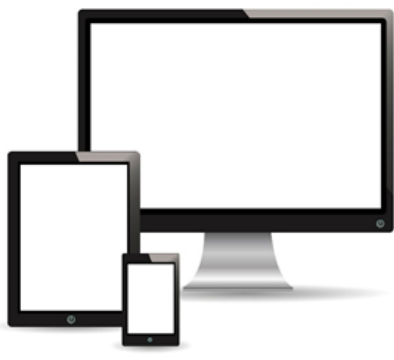

Monitoring device: Smartphone, Tablet or PC

Table 1. Normalized force-sensitive resistor sensors activation registered from volunteers standing at different positions on the mat; standing still and on their toes with both feet and standing still and on their toes with one foot.

\begin{tabular}{lllll}
\hline Sensor activation & Both feet $\%$ & & One foot $\%$ \\
& Standing & Toes & Standing & Toes \\
\hline Average & 71.66 & 28.90 & 40.37 & 61.45 \\
Maximum & 100.00 & 43.31 & 22.64 & 27.00 \\
Minimum & 44.00 & 23.70 & 12.09 \\
\hline
\end{tabular}

For every position sensor activation percentage, a correlation analysis with the weight and foot size of the volunteers was performed. Analysis suggests that sensor activation is only moderately impacted by the weight of volunteers, and foot size of volunteers has a low impact on sensor activation. In Table 2 , correlation values for different positions analyzed on the mat are shown.

To calculate the height reached by the user during the vertical jump, when the subject jumps and there is no contact on the mat, the system counts the elapsed time until the subject lands on the mat again (flight time), and with the calculated time the flight-time formula is used $[3,8,9,14,16]$ to predict the height reached. This formula is defined as: Height $=g \Delta t^{2} / 8$, where $g$ is the constant value of gravity force $g=9.81 \mathrm{~m} / \mathrm{s}^{2}$ and $\Delta \mathrm{t}$ is the flight time obtained by the system. Once the height of the vertical jump is obtained, this value is wirelessly sent via Bluetooth to the monitoring device selected by the health care professional.

Table 2. Pearson correlation coefficient values (R values) for different positions analyzed, calculated for weight and foot size of volunteers.

\begin{tabular}{lcc}
\hline Position & Weight & Foot size \\
\hline Both feet & & -.522 \\
Standing & -.684 & -.241 \\
Forefoot & -.411 & \\
One foot & & -.397 \\
Standing & -.447 & -.463 \\
Forefoot & -.394 & \\
\hline
\end{tabular}




\section{Experimental Setup}

Two different experiments were performed. The purpose of the first experiment, in which 38 volunteers participated, was to validate the reliability of the proposed system. For the second experiment, the objective was to compare the effects of different sampling frequencies when calculating the height reached on the jumps, and 15 volunteers participated. The protocol used for each experiment is the same. For the first experiment, data are directly processed by the microcontroller and the predicted value is sent to the selected monitoring station. In the second experiment, raw data are obtained to perform an offline downsampling to analyze the effects of different sampling frequencies on the predicted result.

Researchers asked for assistance from a sports and fitness center to recruit volunteers who attended the center regularly for physical training. Researchers visited this center with all the necessary equipment for the implementation of the protocols, and installed it in an area specified by the managers of the center. No specific physical attributes were required from the volunteers, as these characteristics should not affect the performance of the system. Researchers approached people at the center, explained the purpose of the study, and politely asked for their collaboration on the protocols if they were available at any given moment. Before starting any trial, volunteers were asked if they had any kind of injury that could affect their physical integrity when performing the protocol, and if so, the trials would not proceed. Every volunteer gave their written consent for the performance of the proposed protocol. The countermovement jump (CMJ) and countermovement jump with arm swing (CMJAS) techniques were selected for this protocol. These jumping techniques are commonly used as a measure to assess the overall force and explosive power of the lower body muscles on a person [26], and it is considered as the most reliable jumping test for this purpose [27]. By adding an arm swing to the CMJ, with the proper technique, the height reached by the person is increased around one-third and up to two-thirds [28-30], which increases the dynamic range of data obtained.

In the proposed protocol, volunteers were asked to stand on a marker placed on the center of the mat and perform 3 medium-to-maximal effort CMJs, with their hands fixed at the waist, with 5 to 10 seconds rest between trials. This technique is depicted in Figure 5. After these jumps, the volunteers were asked to perform CMJAS this time, and following the same scheme. This technique is depicted in Figure 6. As a reference system, all trials were recorded on video with a high-speed camera $(120 \mathrm{fps})$. The camera was placed $1.3 \mathrm{~m}$ away from the mat, perpendicular to the sagittal plane of the volunteer and 20 $\mathrm{cm}$ above the ground, held by a tripod. The setup for this protocol is depicted in Figure 7.

To measure height reached by the subject with the video reference, the takeoff and landing frames were selected manually like in the studies of Balsalobre-Fernandez et al [11,22], and height was calculated by using the elapsed time between the frames using the flight-time formula.

Figure 5. Countermovement jump technique, step by step.

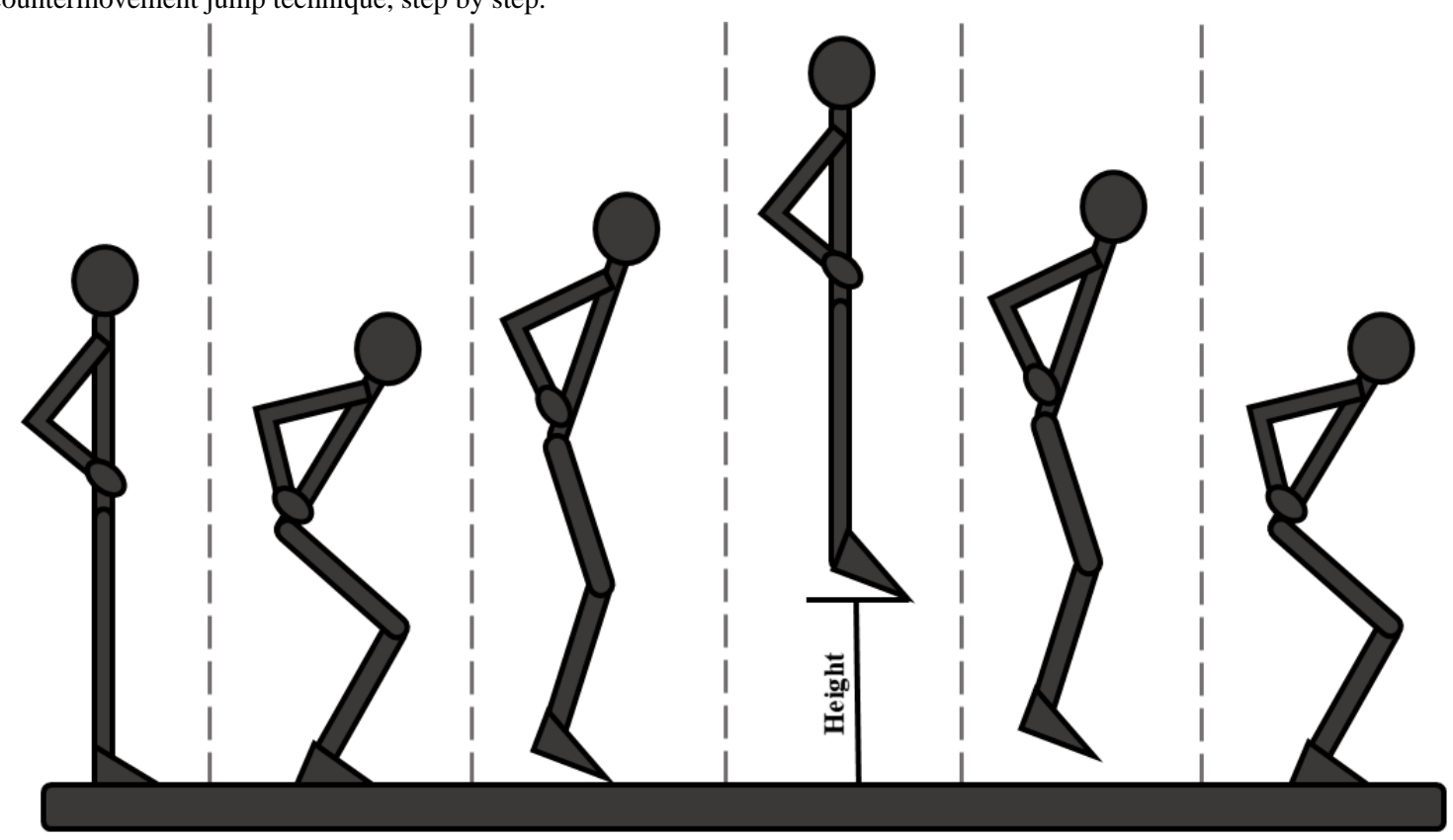


Figure 6. Countermovement jump with arm swing technique, step by step.

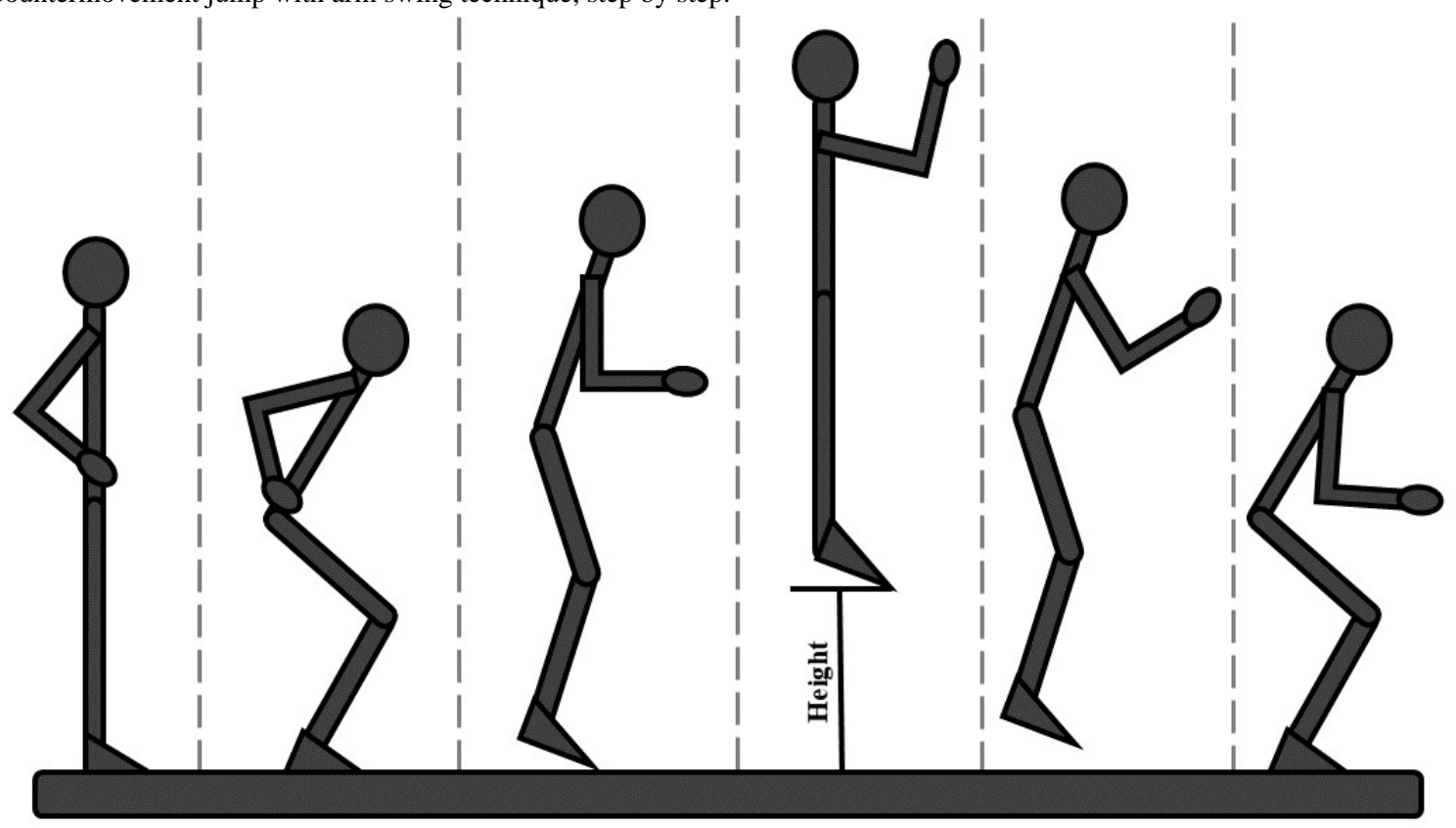

Figure 7. Experimental setup used for proposed experiments.

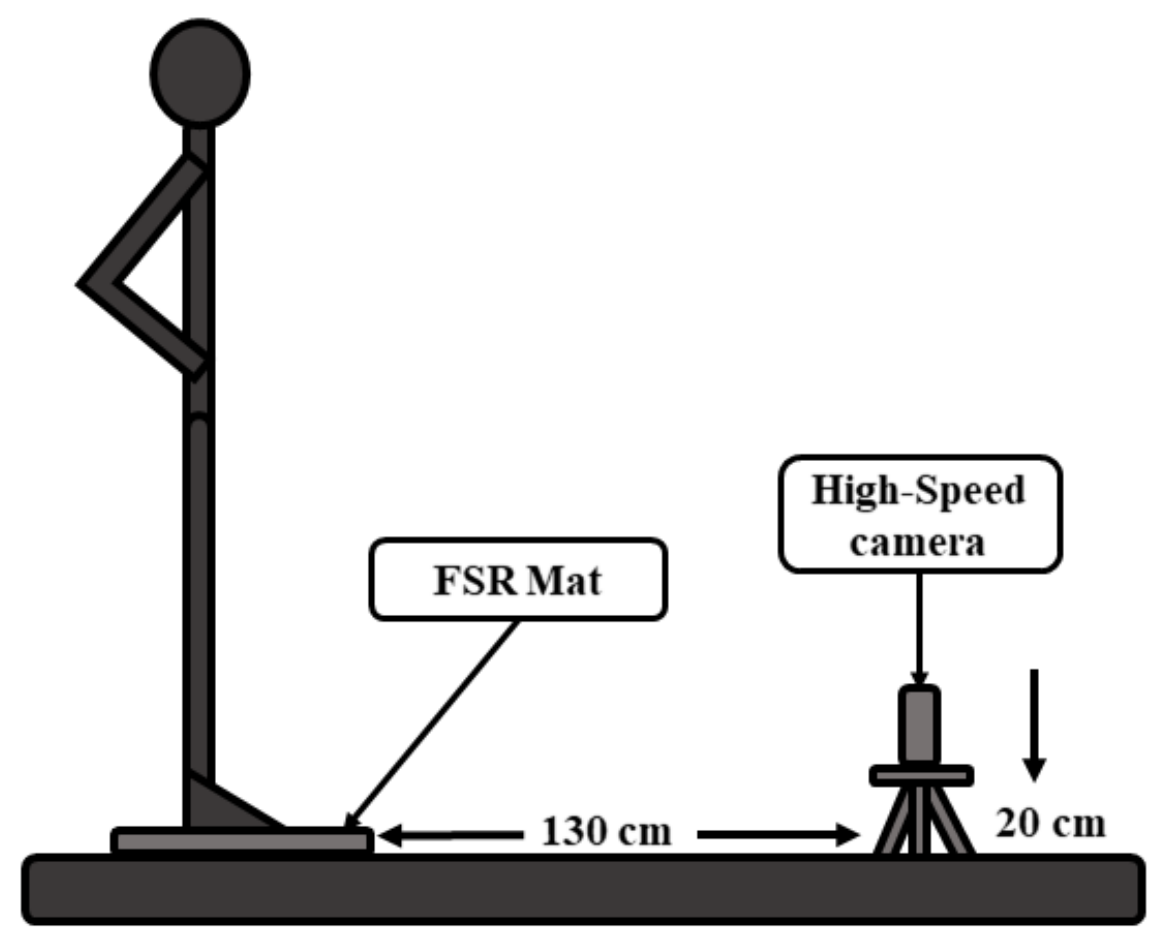

\section{Results}

\section{First Experiment: System Validation}

For the first experiment, a total of 228 jumps (114 CMJs and 114 CMJASs) were performed for each, the proposed system and the video reference. An example of the recorded jumps is shown in Figure 8.
To analyze the proposed system performance, mean relative error (MRE) and mean absolute error (MAE) were calculated for the overall jump trials and for each technique, CMJ and CMJAS. The MRE obtained from all 228 trials was $1.98 \%$. For CMJ and CMJAS, relative errors were $2.17 \%$ and $1.78 \%$, respectively. MAE obtained from all jump trials was $0.38 \mathrm{~cm}$, and for CMJ and CMJAS, the errors obtained were $0.34 \mathrm{~cm}$ and $0.42 \mathrm{~cm}$, respectively. These results are summarized in Table 3 . 
Figure 8. Two volunteers performing the proposed protocol showing the different phases of the jumps: takeoff frame, maximum-height frame, and landing frame.

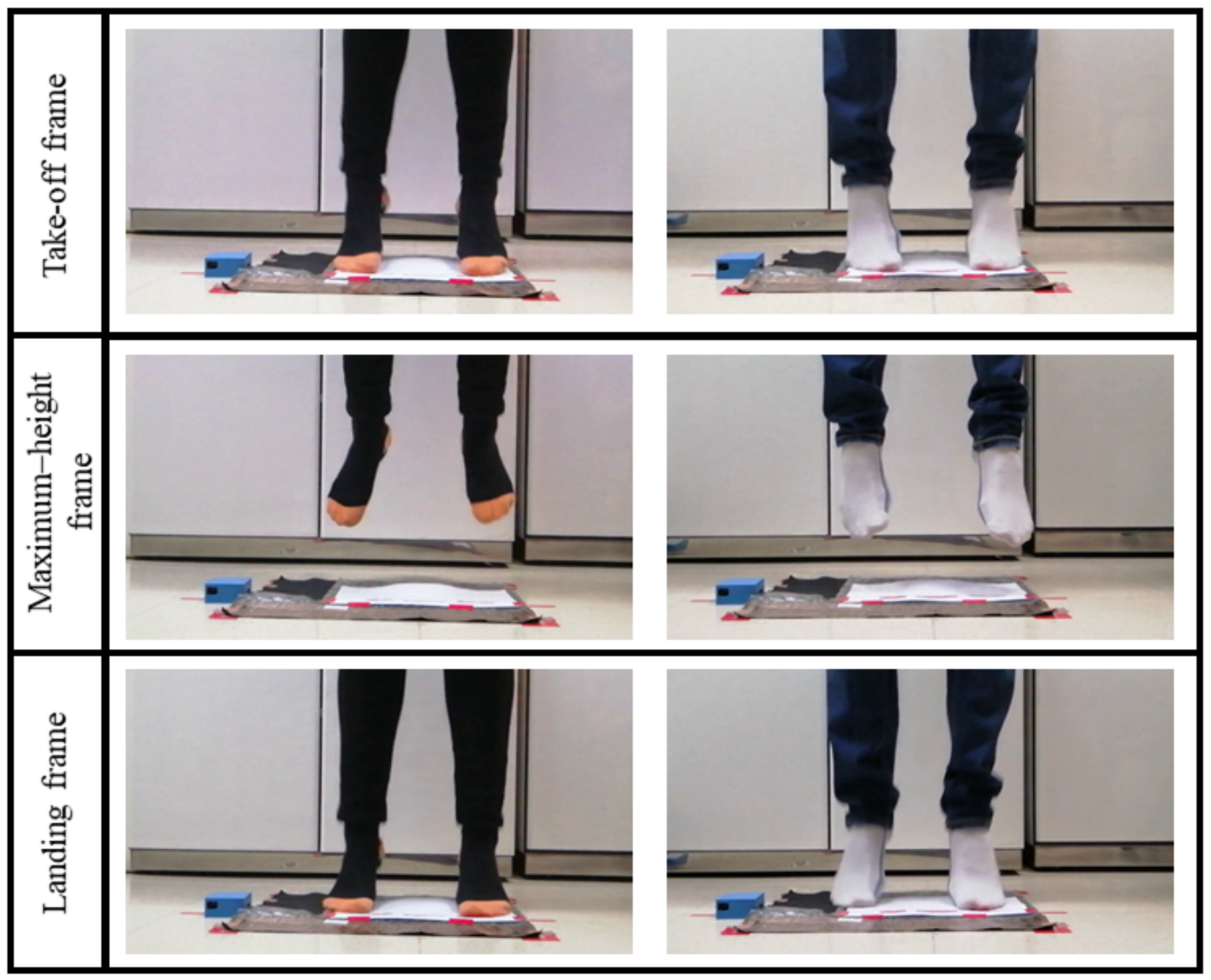

Table 3. Mean absolute error and mean relative error values for overall jump trials, only countermovement jump, and only countermovement jump with arm swing trials.

\begin{tabular}{lll}
\hline Trials & $\operatorname{MAE}^{\mathrm{a}}(\mathrm{cm})$ & MRE $^{\mathrm{b}}(\%)$ \\
\hline Overall & 0.38 & 1.98 \\
$\mathrm{CMJ}^{\mathrm{c}}$ & 0.34 & 2.17 \\
$\mathrm{CMJAS}^{\mathrm{d}}$ & 0.42 & 1.78 \\
\hline
\end{tabular}

${ }^{\mathrm{a}}$ MAE: mean absolute error.

${ }^{b}$ MRE: mean relative error.

${ }^{\mathrm{c}} \mathrm{CMJ}$ : countermovement jump.

${ }^{\mathrm{d}}$ CMJAS: countermovement jump with arm swing.

Correlation and Bland-Altman analyses were performed from data obtained and are shown in Figure 9 and Figure 10, respectively. Correlation analysis shows a coefficient of determination of $R^{2}=.996$. These analyses demonstrate that the proposed system not only has a high correlation, but it shows that the difference of the two paired measurements is really low, which means that both methods produce systematically the same results. 
Figure 9. Correlation graph comparing both measuring methods for the first experiment, showing a coefficient of determination of $R^{2}=.996$.

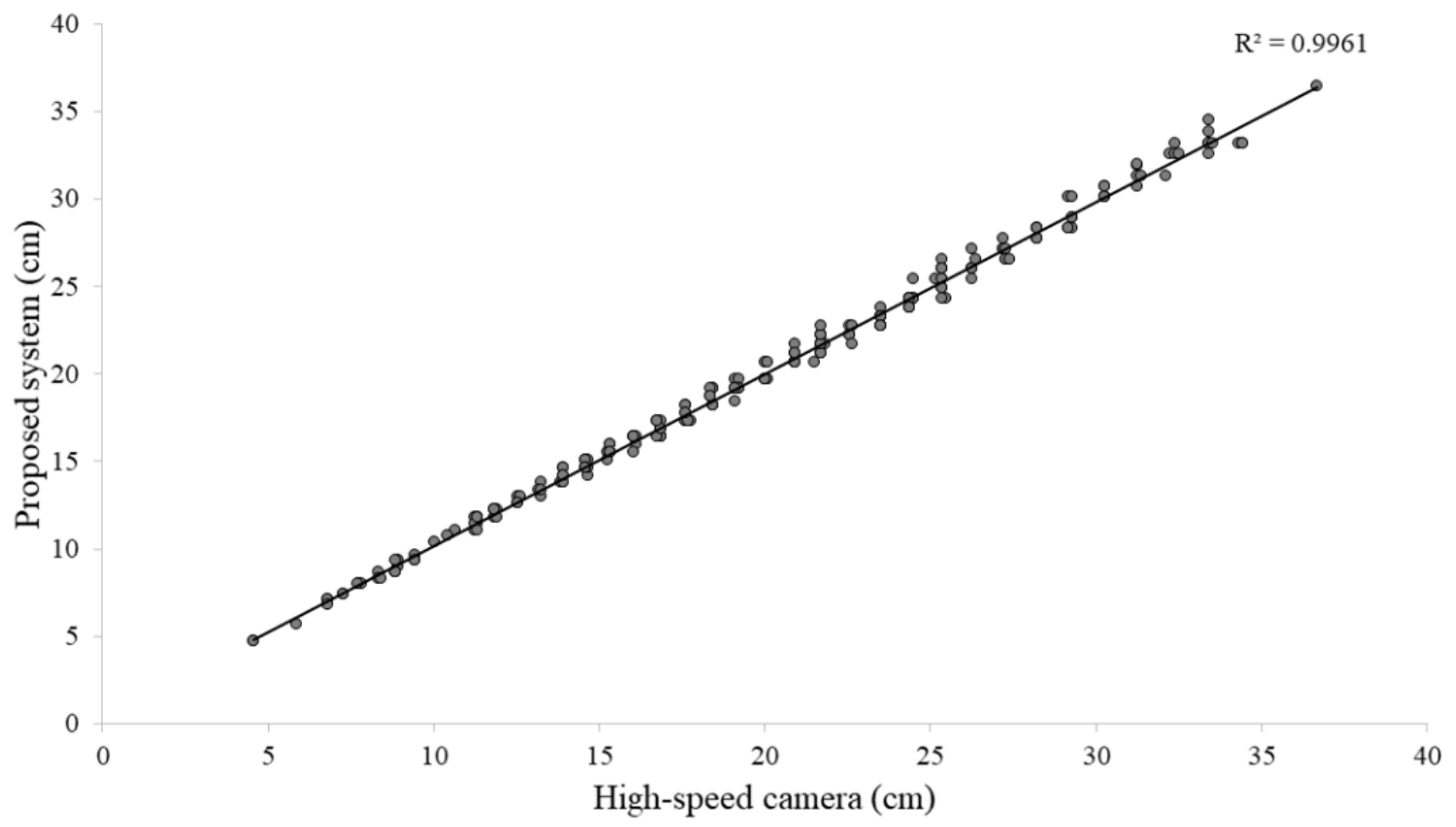

Figure 10. Bland-Altman plot of both measuring methods: countermovement jump depicted by dark gray points and countermovement jump with arm swing depicted by light gray points.

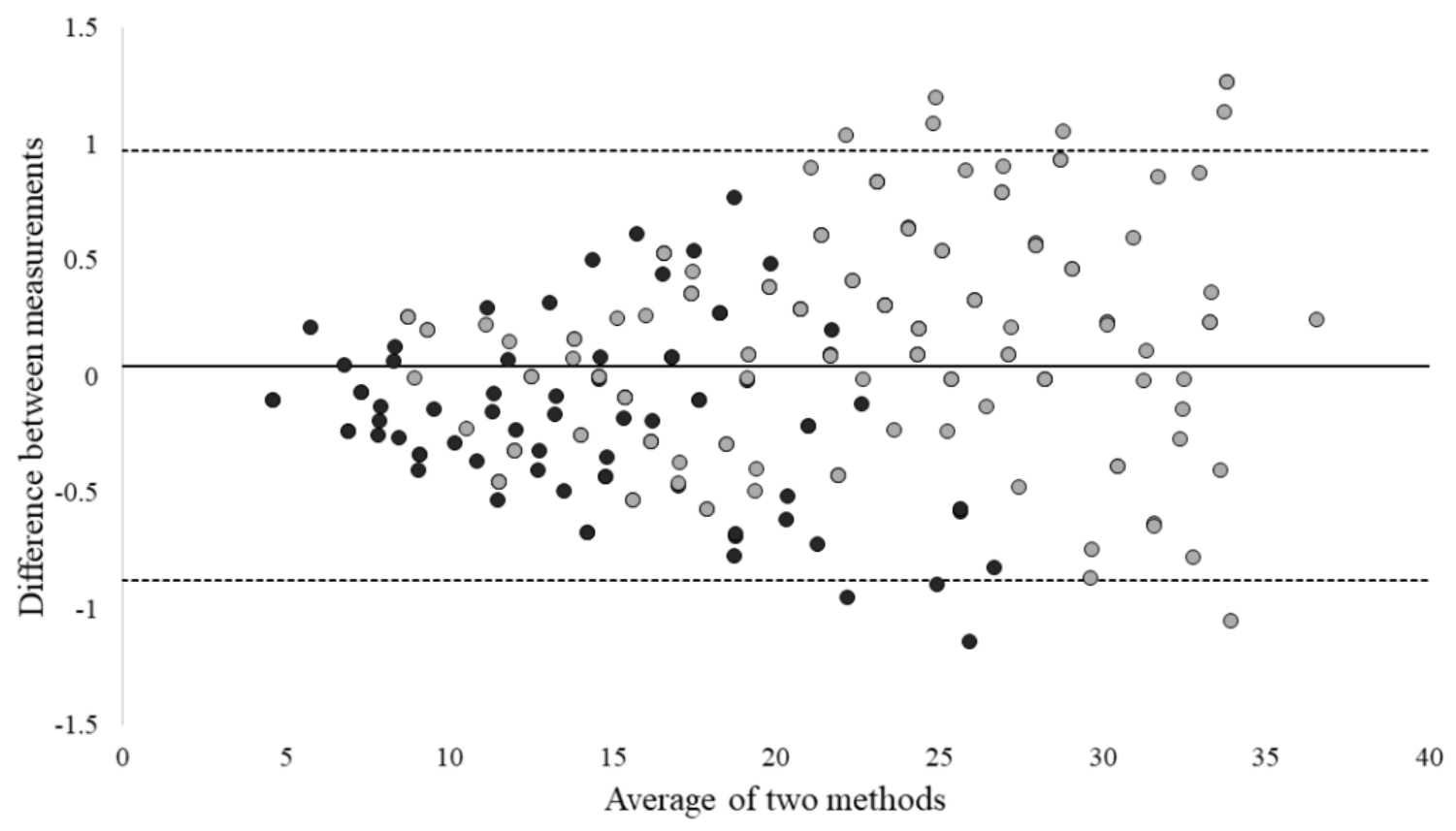

In Figure 11, the normalized MAE and MRE are shown for different ranges of jump heights reached. By analyzing the different ranges of height reached by the volunteers, which are $<10 \mathrm{~cm}, 10$ to $20 \mathrm{~cm}, 20$ to $30 \mathrm{~cm}$, and $>30 \mathrm{~cm}$, MREs obtained were $2.38 \%, 2.07 \%, 1.90 \%$, and $1.54 \%$, respectively. MAE obtained were $0.18 \mathrm{~cm}, 0.31 \mathrm{~cm}, 0.46 \mathrm{~cm}$, and $0.50 \mathrm{~cm}$, respectively. From this data, no significant difference can be found. However, it can be noticed that MAE increases as jump height increases, while MRE decreases.
Figure 12 shows the charts with distribution of the heights reached by the volunteers when performing the jump trials. For CMJ, no volunteer was able to surpass the $30 \mathrm{~cm}$ height. However, by adding the arm swing, $22 \%$ of the volunteers surpassed the $30 \mathrm{~cm}$ height. Also, for CMJAS trials, $47 \%$ of the subjects reached a height ranging from 20 to $30 \mathrm{~cm}$, compared with CMJ, in which only $31 \%$ of the subjects reached this height. 
Figure 11. Normalized mean absolute error and mean relative error, divided in different ranges of height reached during vertical jump.

$\square$ Absolute error $\square$ Relative error

0.6

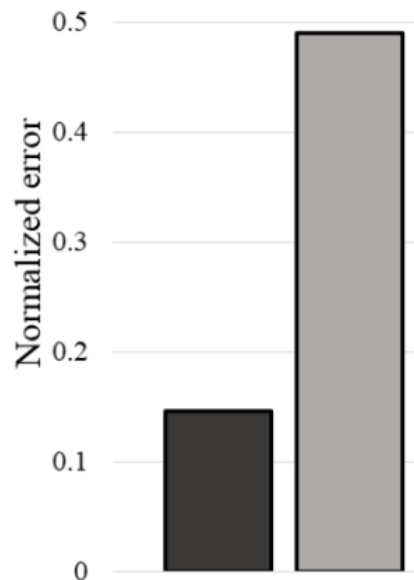

$<10 \mathrm{~cm}$

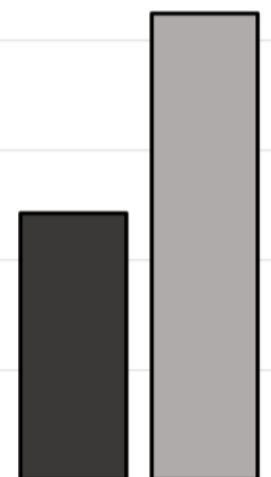

$10-20 \mathrm{~cm}$

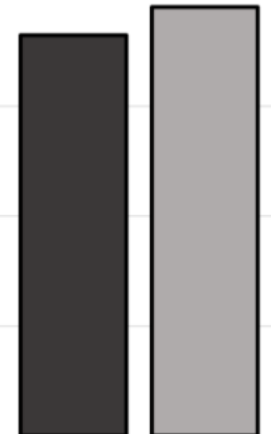

$20-30 \mathrm{~cm}$

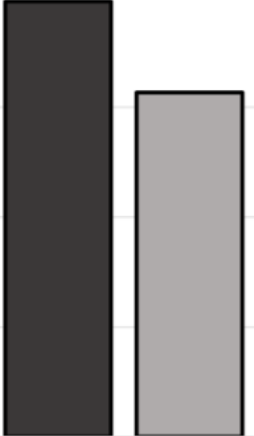

$>30 \mathrm{~cm}$

Jump height (Reference)

Figure 12. Distribution of different height ranges reached by the users. Overall trials, only countermovement jump trials, and only countermovement jump with arm swing trials are shown.

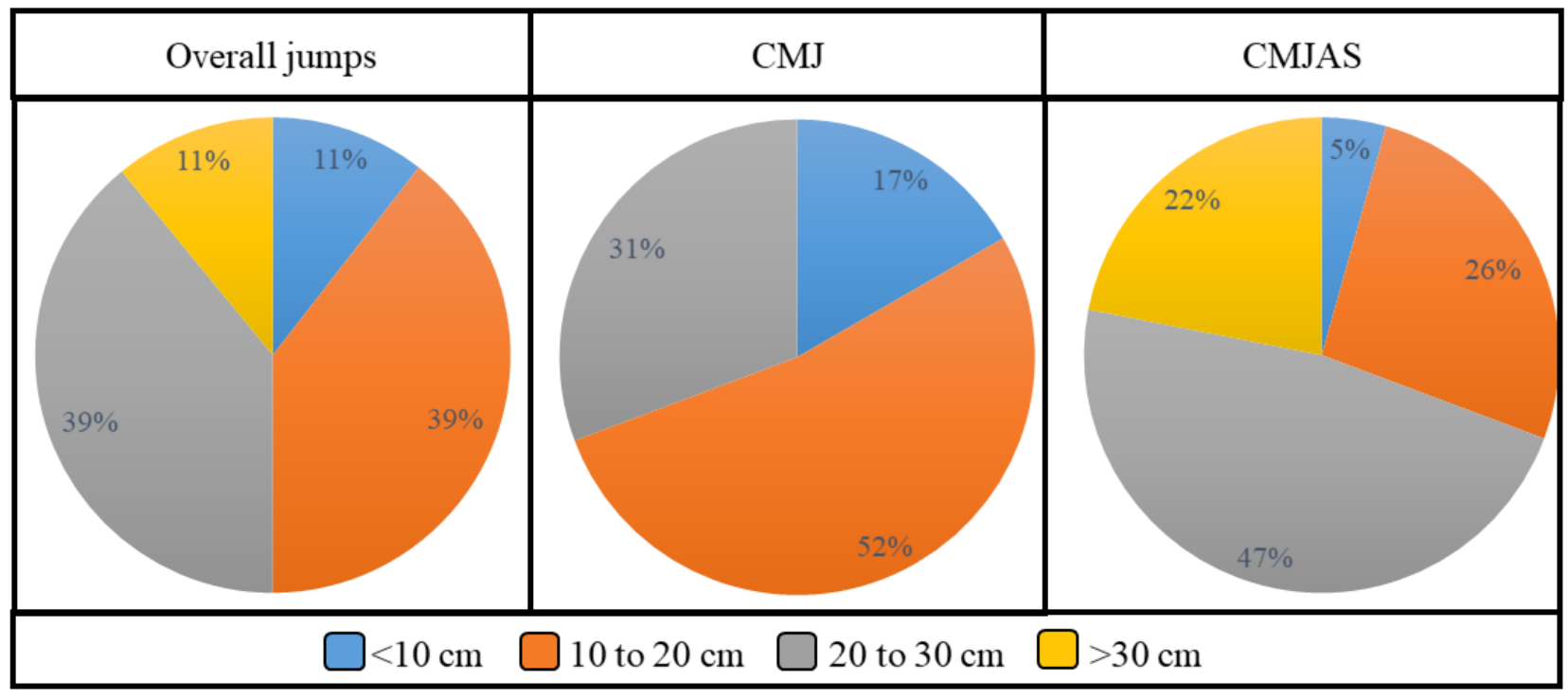

\section{Second Experiment: Sampling Frequencies Comparison}

In this experiment, the effects of different sampling frequencies were analyzed. Raw data from the system was obtained for a total of 90 jumps (45 CMJs and 45 CMJASs). An offline emulation of different sampling frequencies was performed through downsampling of this raw data. This means samples are removed to emulate a slower sampling frequency. With this method, and with the base sampling period of $5 \mathrm{~ms}$ from the system, $200 \mathrm{~Hz}, 100 \mathrm{~Hz}, 66.6 \mathrm{~Hz}, 50 \mathrm{~Hz}, 40 \mathrm{~Hz}, 33.3 \mathrm{~Hz}, 28.5$ $\mathrm{Hz}, 25 \mathrm{~Hz}, 22.2 \mathrm{~Hz}$, and $20 \mathrm{~Hz}$ frequencies were emulated.

Similar to the first experiment, the error was calculated using the high-speed camera as reference. For this analysis, only MRE was obtained for each sampling frequency to assess which frequencies are able to maintain a relative error below $5 \%$.
Results show that sampling frequencies of $200 \mathrm{~Hz}, 100 \mathrm{~Hz}$, and $66.6 \mathrm{~Hz}$ have similar performance, with relative errors of $1.88 \%$, $2.22 \%$, and $2.88 \%$, respectively. However, the maximum error among the 90 trials increases considerably between these frequencies, with maximum errors of $5.27 \%, 7.02 \%$, and $8.25 \%$ for each respective frequency. Sampling frequencies of $50 \mathrm{~Hz}$, $40 \mathrm{~Hz}$, and $33.3 \mathrm{~Hz}$ also show good performance regarding the relative error, which is maintained below $5 \%$ for the 3 cases, but the maximum relative error found in these 3 frequencies is considerably higher than the found in the previous set.

In Table 4, MRE and maximum and minimum relative errors found among trials for the different sampling frequencies are shown. With slower sampling frequencies, MRE increases exponentially as shown in Figure 13, which suggests that sampling frequencies equal to or below $28.5 \mathrm{~Hz}$ are not reliable enough to maintain MRE below 5\%. Also, sampling frequencies 
slower than $50 \mathrm{~Hz}$ and $33.3 \mathrm{~Hz}$ show maximum relative error among trials higher than $10 \%$ and $20 \%$, respectively.

Table 5 shows how MRE is distributed in different ranges. Only $200 \mathrm{~Hz}$ and $100 \mathrm{~Hz}$ sampling frequencies are able to maintain $95 \%$ of their results within $5 \%$ of relative error. Also, sampling

Figure 13. Mean relative error obtained for each proposed sampling frequency. As sampling frequency decreases, relative error increases exponentially.

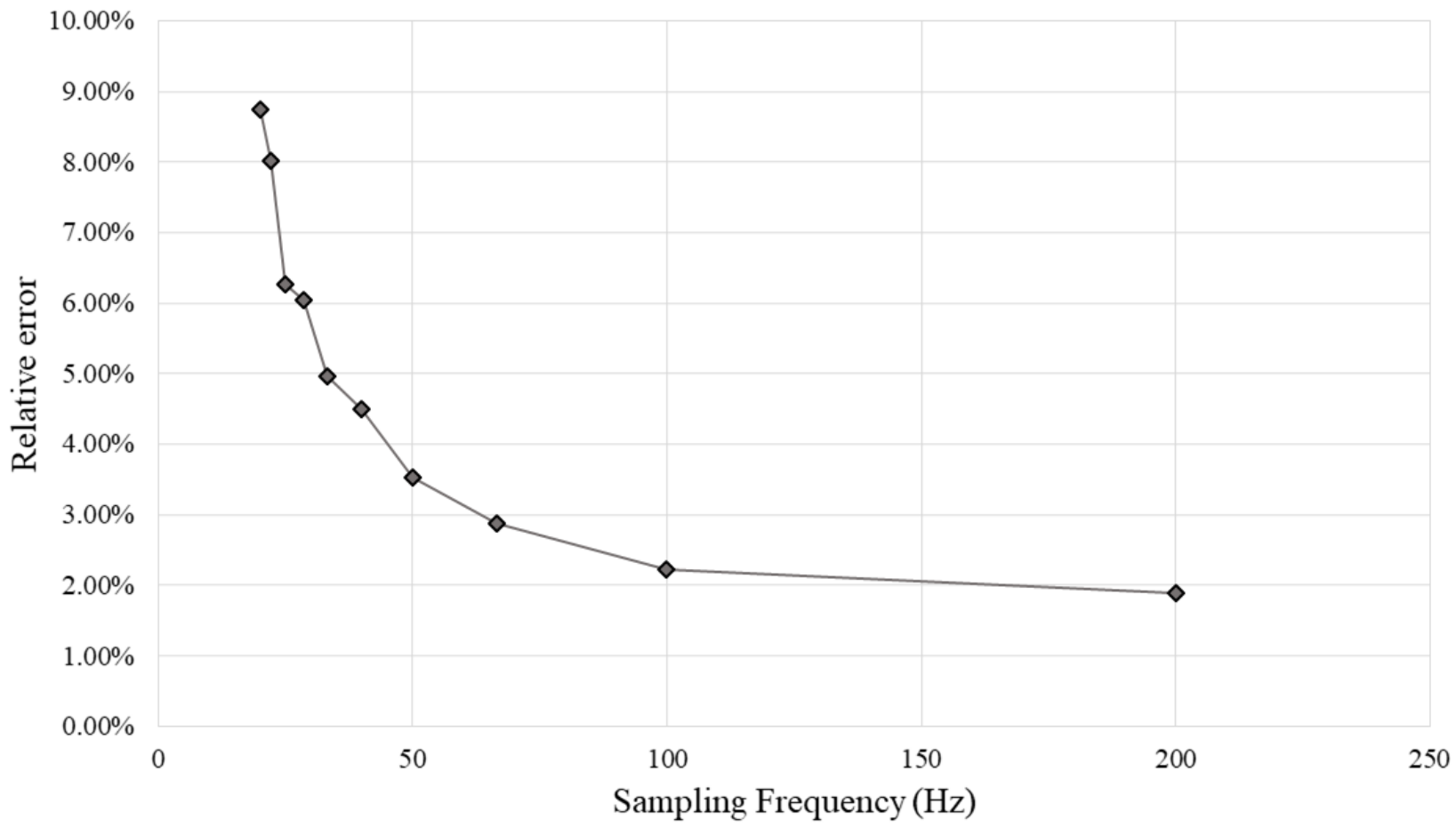

Table 4. Mean relative error and maximum and minimum relative errors obtained from all 90 trials for each sampling frequency analyzed.

\begin{tabular}{|c|c|c|c|c|c|c|c|c|c|c|}
\hline \multirow{2}{*}{$\begin{array}{l}\text { Relative } \\
\text { error }\end{array}$} & \multicolumn{10}{|c|}{ Sampling periods/frequencies } \\
\hline & $\begin{array}{l}5 \mathrm{~ms}, 200 \\
\mathrm{~Hz}\end{array}$ & $\begin{array}{l}10 \mathrm{~ms}, 100 \\
\mathrm{~Hz}\end{array}$ & $\begin{array}{l}15 \mathrm{~ms}, 66.6 \\
\mathrm{~Hz}\end{array}$ & $\begin{array}{l}20 \mathrm{~ms}, 50 \\
\mathrm{~Hz}\end{array}$ & $\begin{array}{l}25 \mathrm{~ms}, 40 \\
\mathrm{~Hz}\end{array}$ & $\begin{array}{l}30 \mathrm{~ms}, 33.3 \\
\mathrm{~Hz}\end{array}$ & $\begin{array}{l}35 \mathrm{~ms}, 28.5 \\
\mathrm{~Hz}\end{array}$ & $\begin{array}{l}40 \mathrm{~ms}, 25 \\
\mathrm{~Hz}\end{array}$ & $\begin{array}{l}45 \mathrm{~ms}, 22.2 \\
\mathrm{~Hz}\end{array}$ & $\begin{array}{l}50 \mathrm{~ms}, 20 \\
\mathrm{~Hz}\end{array}$ \\
\hline $\mathrm{MRE}^{\mathrm{a}}$ & 1.88 & 2.22 & 2.88 & 3.52 & 4.50 & 4.97 & 6.04 & 6.27 & 8.02 & 8.75 \\
\hline $\mathrm{MAX}^{\mathrm{b}}$ & 5.27 & 7.02 & 8.25 & 9.73 & 14.25 & 14.25 & 21.30 & 19.73 & 28.39 & 32.11 \\
\hline $\mathrm{MIN}^{\mathrm{c}}$ & 0 & 0 & 0 & 0 & 0.70 & 0 & 0 & 0 & 0 & 0.73 \\
\hline
\end{tabular}

${ }^{\mathrm{a}}$ MRE: mean relative error.

${ }^{\mathrm{b}} \mathrm{MAX}$ : maximum relative error.

${ }^{\mathrm{c}} \mathrm{MIN}$ : minimum relative error.

Table 5. Percentage of trials whose relative error is within the ranges of 5\% or less, higher than $5 \%$ and lower than $15 \%$, and higher than $15 \%$.

\begin{tabular}{lllllllllll}
\hline Relative error & \multicolumn{2}{l}{ Sampling periods/frequencies } & & & & & \\
& $5 \mathrm{~ms}, 200$ & $10 \mathrm{~ms}$, & $15 \mathrm{~ms}, 66.6$ & $20 \mathrm{~ms}, 50$ & $25 \mathrm{~ms}, 40$ & $30 \mathrm{~ms}, 33.3$ & $35 \mathrm{~ms}, 28.5$ & $40 \mathrm{~ms}, 25$ & $45 \mathrm{~ms}, 22.2$ & $50 \mathrm{~ms}, 20$ \\
& $\mathrm{~Hz}$ & $100 \mathrm{~Hz}$ & $\mathrm{~Hz}$ & $\mathrm{~Hz}$ & $\mathrm{~Hz}$ & $\mathrm{~Hz}$ & $\mathrm{~Hz}$ & $\mathrm{~Hz}$ & $\mathrm{~Hz}$ \\
\hline $\mathrm{RE}^{\mathrm{a}} \leq 5 \%$ & 98.89 & 94.44 & 87.78 & 76.67 & 58.89 & 57.78 & 48.89 & 52.22 & 35.56 & 27.78 \\
$\mathrm{RE} 5 \%$ to $15 \%$ & 1.11 & 5.56 & 12.22 & 23.33 & 41.11 & 42.22 & 47.78 & 43.33 & 54.44 & 57.78 \\
$\mathrm{RE}>15 \%$ & 0 & 0 & 0 & 0 & 0 & 0 & 3.33 & 4.44 & 10 & 14.44 \\
\hline
\end{tabular}

${ }^{\mathrm{a}} \mathrm{RE}$ : relative error. 
When MRE is obtained from different jump heights $(\leq 10 \mathrm{~cm}$, 10 to $20 \mathrm{~cm}, 20$ to $30 \mathrm{~cm}$, and $>30 \mathrm{~cm}$ ) at each sampling frequency, the relevance of a proper sampling frequency when calculating height reached for small jumps $(<20 \mathrm{~cm})$ is observed. When using sampling frequencies slower than $50 \mathrm{~Hz}$, MRE obtained from these small jumps is always higher than $5 \%$, and for the slowest sampling frequency, MRE reaches a value of $21.50 \%$ for jump heights smaller than $10 \mathrm{~cm}$. For higher jump heights, an increase in MRE (>5\%) is noticeable for sampling frequencies slower than $40 \mathrm{~Hz}$, reaching a value of up to $9.15 \%$ for the slowest sampling frequency. A summary of these results is shown in Table 6.

Table 6. Mean relative error obtained from each of the analyzed sampling frequencies for different ranges of height reached during the vertical jump.

\begin{tabular}{|c|c|c|c|c|c|c|c|c|c|c|}
\hline \multirow[t]{2}{*}{ Jump height } & \multicolumn{10}{|c|}{ Sampling periods/frequencies } \\
\hline & $5 \mathrm{~ms}, 200$ & $10 \mathrm{~ms}, 100$ & $15 \mathrm{~ms}, 66.6$ & $20 \mathrm{~ms}, 50$ & $25 \mathrm{~ms}, 40$ & $30 \mathrm{~ms}, 33.3$ & $35 \mathrm{~ms}, 28.5$ & $40 \mathrm{~ms}, 25$ & $45 \mathrm{~ms}, 22.2$ & $50 \mathrm{~ms}, 20$ \\
\hline$<10 \mathrm{~cm}$ & 1.31 & 2.29 & 4.33 & 2.62 & 5.28 & 5.09 & 5.40 & 6.29 & 10.77 & 21.50 \\
\hline $10-20 \mathrm{~cm}$ & 2.23 & 2.40 & 2.89 & 4.29 & 5.21 & 5.45 & 7.55 & 8.60 & 9.65 & 8.42 \\
\hline $20-30 \mathrm{~cm}$ & 1.91 & 2.31 & 3.02 & 3.55 & 4.62 & 5.29 & 5.06 & 6.12 & 6.96 & 9.15 \\
\hline$>30 \mathrm{~cm}$ & 1.60 & 1.95 & 2.50 & 2.92 & 3.63 & 4.17 & 5.83 & 4.34 & 7.32 & 6.85 \\
\hline
\end{tabular}

\section{Discussion}

\section{Principal Findings}

The vertical jump is a test commonly used by health care professionals to assess strength in the lower limb muscles of a subject. Although this test is widely used for strength assessment among athletes, relevant information can be obtained from people with no relevant sports background.

An important point to highlight about the system developed in this study is its low price. The total for components used in construction is approximately US \$40. In comparison with commercially available devices, this developed system is significantly more affordable. Among the devices commonly used on medical and sports fields to measure vertical jump height are the vertical jump test mat (Gill Athletics) [31], Just Jump system (Perform Better) [10,32], Vertec device (Gill Athletics) [10,33], electronic vertical jump tester (Gill Athletics) [34], Optojump testing (Perform Better) [12,35], and bilateral force plate (Hawkin Dynamics) [18,36]. Our proposed system will have to pass through different standards and certifications (like ISO standards [37]) before it can be considered as a standard medical device. Table 7 shows a comparison of prices between commercially available devices and the system proposed here. Prices of the commercially available devices are listed as found at the moment of writing this article.

Table 7. Comparison of prices between commercially available devices and the system developed in this study.

\begin{tabular}{ll}
\hline Device for vertical jump measurement & Price \$ \\
\hline Proposal from this study (estimated price of components) & 40 \\
Vertical jump test mat (Gill Athletics) [31] & 360 \\
Just Jump System (Perform Better) [32] & 629 \\
Vertec device (Gill Athletics) [33] & 760 \\
Electronic vertical jump tester (Gill Athletics) [34] & 2925 \\
Optojump testing (Perform Better) [35] & 3804 \\
Bilateral force plate (Hawkin Dynamics) [36] & 5000 \\
\hline
\end{tabular}

Throughout data capturing in both experiments, some important points can be highlighted. Despite the advantages that the proposed system and reference device offer, both have an inherent error due to their sampling frequency (more specifically due to their sampling period). The proposed system has a sampling frequency of $200 \mathrm{~Hz}$, and thus the sampling period is $5 \mathrm{~ms}$. Likewise, the reference device has a sampling frequency of $120 \mathrm{~Hz}$ and a sampling period of $8.3 \mathrm{~ms}$. This means that every sampling period each device updates its readings, which implies an uncertainty of the sampling period between data updates. In other words, there is an inherent uncertainty in the system during the takeoff and landing phases of the jump, time span that is used to calculate height reached. From both phases, the proposed system has a total uncertainty of $10 \mathrm{~ms}$, while the reference device has a total uncertainty of $16.6 \mathrm{~ms}$. This inherent error is characteristic of electronic devices and directly related to their sampling frequency. Nonelectronic methods for jump height measuring lack of this inherent error, but as stated before, these methods tend to overestimate obtained measurements and are less precise.

Regarding the high-speed camera used as a reference device, when the recorded videos were analyzed, the ease of selecting the correct frames depended on the correct technique execution of the volunteer: taking off from both forefeet at the same time during the takeoff phase and landing with both forefeet at the same time during the landing phase. This was the ideal technique execution. On the other hand, some volunteers either took off or landed with only one forefoot and not with the same foot in 
some occasions. In such cases, it was harder to select the takeoff and landing frames. This is difficult for volunteers to control without long-term training in the proper technique.

On the proposed protocol, the inclusion of two different jump techniques proved to be useful in order to increase the dynamic range of the data. The difference between the CMJ and CMJAS was significant. The addition of an arm swing increased jump height an average of $44.84 \%$ in the first experiment and $34.86 \%$ in the second experiment.

\section{Limitations}

One of the main limitations of the developed system was its sampling frequency. Although the microcontroller used had a high CPU frequency, the sampling frequency was limited because of the number of operations needed for a full scan of the mat ( 16 rows and 16 columns, a scan of 256 individual cells) and number of operations this implies. Another limitation was the total sensing area of the system of $30 \times 30 \mathrm{~cm}$. Although no volunteer reported discomfort, the total area limits the stance of volunteers; in addition, the landing phase of every jump trial must be performed in a controlled manner, so the volunteer lands inside this area.

These limitations can be solved in future versions of the mat. The design of the mat can be modified to increase its total sensing area and the size of each row and column, so in this way with fewer number of rows and columns the same sensing area could be achieved, thus increasing the sampling frequency of the system. However, this would diminish the resolution of the system.

\section{Conclusions}

In this study, a novel low-cost system for measurement of the jump height is proposed. Two experiments were performed - one to validate the system and the other to assess the effects of different sampling frequencies.

When evaluating the performance of the proposed system in the first experiment, results show that with the proposed sampling frequency of $200 \mathrm{~Hz}$ relative error for all of the 228 jump trials is maintained below $5 \%$. In the second experiment, with sampling frequencies of $200 \mathrm{~Hz}$ and $100 \mathrm{~Hz}$, relative error is maintained below $5 \%$ for $98.89 \%$ and $94.44 \%$ of the jump trials, respectively.

The flight-time formula is a widely used, validated method to calculate height reached during vertical jumps. A high-speed camera as reference device has been used in related studies along with the flight-time formula, proving to be a reliable tool. Our first experiment showed through correlation and Bland-Altman analyses that the proposed system and a high-speed camera reference device produced systematically similar results when calculating jump height.

Our second experiment concluded that $200 \mathrm{~Hz}$ and $100 \mathrm{~Hz}$ sampling frequencies have similar performance, and both frequencies are reliable when calculating jump height using the flight-time formula. This implies that if access to hardware capable of processing data at $200 \mathrm{~Hz}$ were limited, hardware capable of processing data to at least $100 \mathrm{~Hz}$ could offer similar results. However, if higher sampling frequencies are available, they should be used.

These results demonstrate that the proposed system is as reliable as a commercially available device, and the selected sampling frequency of $200 \mathrm{~Hz}$ is reliable for obtaining relative errors below $5 \%$ for at least $95 \%$ of the jump trials. The proposed system offers an alternative for health care professionals to use a mobile monitoring station of their choice, and its price is more affordable than commercially available devices.

\section{Acknowledgments}

This research was funded by grant Programa Operativo FEDER Construyendo Europa desde Aragon T49_20R from the European Union and Gobierno de Aragón, grant UZCUD2019-TEC-02 from the Universidad de Zaragoza and Centro Universitario de la Defensa de Zaragoza, and grant 709365 from the Consejo Nacional de Ciencia y Tecnología.

\section{Conflicts of Interest}

None declared.

\section{References}

1. Rodrigues C, Correia M, Abrantes J, Nadal J, Benedetti Rodrigues MA. Consistency of surface electromyography assessment at lower limb selected muscles during vertical countermovement. Annu Int Conf IEEE Eng Med Biol Soc 2017 Jul;2017:402-405. [doi: 10.1109/EMBC.2017.8036847] [Medline: 29059895]

2. Garcia M, Guzmán L, Valencia J, Henao V. Portable measurement system of vertical jump using an inertial measurement unit and pressure sensors. 2016 Presented at: 2016 XXI Symposium on Signal Processing, Images and Artificial Vision (STSIVA); 2016; Bucaramanga p. 1-5. [doi: 10.1109/stsiva.2016.7743299]

3. Drazan J, Danielsen H, Vercelletto M, Loya A, Davis J, Eglash R. A case study for integrated STEM outreach in an urban setting using a do-it-yourself vertical jump measurement platform. Annu Int Conf IEEE Eng Med Biol Soc 2016 Aug;2016:3027-3030. [doi: 10.1109/EMBC.2016.7591367] [Medline: 28268950]

4. Casartelli N, Müller R, Maffiuletti N. Validity and reliability of the Myotest accelerometric system for the assessment of vertical jump height. J Strength Cond Res 2010 Nov;24(11):3186-3193. [doi: 10.1519/JSC.0b013e3181d8595c] [Medline: 20940642] 
5. Mijailovic N, Radakovic R, Peulic A, Milankovic I, Filipovic N. Using force plate, computer simulation and image alignment in jumping analysis. 2015 Presented at: 2015 IEEE 15th International Conference on Bioinformatics and Bioengineering (BIBE); 2015; Belgrade p. 1-4. [doi: 10.1109/bibe.2015.7367672]

6. Yahya U, Senanayake S, Naim A. Intelligent integrated wearable sensing mechanism for vertical jump height prediction in female netball players. 2017 Presented at: 2017 Eleventh International Conference on Sensing Technology (ICST); 2017; Sydney p. 1-7. [doi: 10.1109/icsenst.2017.8304483]

7. García-Ramos A, Štirn I, Padial P, Argüelles-Cienfuegos J, De la Fuente B, Strojnik V, et al. Predicting vertical jump height from bar velocity. J Sports Sci Med 2015 Jun;14(2):256-262 [FREE Full text] [Medline: 25983572]

8. Howard R, Conway R, Harrison A. Estimation of force during vertical jumps using body fixed accelerometers. 2014 Presented at: 25th IET Irish Signals \& Systems Conference 2014 and 2014 China-Ireland International Conference on Information and Communications Technologies (ISSC 2014/CIICT 2014); 2014; Limerick p. 102-107. [doi:

$\underline{10.1049 / \mathrm{cp} .2014 .0667]}$

9. Whitmer T, Fry A, Forsythe C, Andre M, Lane M, Hudy A, et al. Accuracy of a vertical jump contact mat for determining jump height and flight time. J Strength Cond Res 2015 Apr;29(4):877-881. [doi: 10.1519/JSC.0000000000000542] [Medline: 24852256]

10. Nuzzo J, Anning J, Scharfenberg J. The reliability of three devices used for measuring vertical jump height. J Strength Cond Res 2011 Sep;25(9):2580-2590. [doi: 10.1519/JSC.0b013e3181fee650] [Medline: 21804426]

11. Balsalobre-Fernández C, Glaister M, Lockey RA. The validity and reliability of an iPhone app for measuring vertical jump performance. J Sports Sci 2015;33(15):1574-1579. [doi: 10.1080/02640414.2014.996184] [Medline: 25555023]

12. Glatthorn J, Gouge S, Nussbaumer S, Stauffacher S, Impellizzeri F, Maffiuletti N. Validity and reliability of Optojump photoelectric cells for estimating vertical jump height. J Strength Cond Res 2011 Feb;25(2):556-560. [doi: 10.1519/JSC.0b013e3181ccb18d] [Medline: 20647944]

13. Bui HT, Farinas M, Fortin A, Comtois A, Leone M. Comparison and analysis of three different methods to evaluate vertical jump height. Clin Physiol Funct Imaging 2015 May;35(3):203-209. [doi: 10.1111/cpf.12148] [Medline: 24690449]

14. Dias J, Dal Pupo J, Reis D, Borges L, Santos S, Moro A, et al. Validity of two methods for estimation of vertical jump height. J Strength Cond Res 2011 Jul;25(7):2034-2039. [doi: 10.1519/JSC.0b013e3181e73f6e] [Medline: 21701288]

15. Kibele A. Possibilities and limitations in the biomechanical analysis of countermovement jumps: a methodological study. J Appl Biomech 1998;14(1):105-117. [doi: 10.1123/jab.14.1.105]

16. Boukhenous S, Attari M. A vertical jumping performance with and without arms swing by using a dynamometric platform. Int Workshop Syst Signal Processing Appl 2011:17-20. [doi: 10.1109/wosspa.2011.5931450]

17. Zihajehzadeh S, Lee TJ, Lee JK, Hoskinson R, Park EJ. Integration of MEMS inertial and pressure sensors for vertical trajectory determination. IEEE Trans Instrum Meas 2015 Mar;64(3):804-814. [doi: 10.1109/tim.2014.2359813]

18. Moir GL. Three different methods of calculating vertical jump height from force platform data in men and women. Meas Phys Educ Exer Sci 2008 Oct 15;12(4):207-218. [doi: 10.1080/10913670802349766]

19. Aragón LF. Evaluation of four vertical jump tests: methodology, reliability, validity, and accuracy. Meas Phys Educ Exer Sci 2000 Dec;4(4):215-228. [doi: 10.1207/s15327841mpee0404 2]

20. Kassim A, Miskon M, Rahim N, Yasuno T. Effectiveness of reference height control system for tripod hopping robot. 2011 Presented at: 2011 4th International Conference on Mechatronics (ICOM); 2011; Kuala Lumpur p. 1-4. [doi: 10.1109/icom.2011.5937123]

21. Leard J, Cirillo M, Katsnelson E, Kimiatek D, Miller T, Trebincevic K, et al. Validity of two alternative systems for measuring vertical jump height. J Strength Cond Res 2007 Nov;21(4):1296-1269. [doi: 10.1519/R-21536.1] [Medline: $\underline{18076265]}$

22. Balsalobre-Fernández C, Tejero-González CM, del Campo-Vecino J, Bavaresco N. The concurrent validity and reliability of a low-cost, high-speed camera-based method for measuring the flight time of vertical jumps. J Strength Cond Res 2014 Feb;28(2):528-533. [doi: 10.1519/JSC.0b013e318299a52e] [Medline: 23689339]

23. Valle-Lopera DA, Castraño-Franco AF, Gallego-Londoño J, Hernández-Valdivieso AM. Test and fabrication of piezoresistive sensors for contact pressure measurement. Rev Fac Ing Univ Antioquia 2017 Mar(82):47-52. [doi: 10.17533/udea.redin.n82a06]

24. Giovanelli D, Farella E. Force sensing resistor and evaluation of technology for wearable body pressure sensing. J Sensors 2016;2016:1-13. [doi: 10.1155/2016/9391850]

25. Medrano-Sanchez C, Igual-Catalan R, Rodriguez-Ontiveros VH, Plaza-Garcia I. Circuit analysis of matrix-like resistor networks for eliminating crosstalk in pressure sensitive mats. IEEE Sensors J 2019 Sep 15;19(18):8027-8036. [doi: 10.1109/jsen.2019.2918592]

26. Young W. Laboratory strength assessment of athletes. 1995. URL: https://www.worldathletics.org/download/ downloadnsa?filename=9daf7a4d-c52b-4956-8453-caa4b378606c.pdf\&urlslug=laboratory-strength-assessment-of-athletes [accessed 2021-03-05]

27. Markovic G, Dizdar D, Jukic I, Cardinale M. Reliability and factorial validity of squat and countermovement jump tests. J Strength Cond Res 2004 Aug;18(3):551-555. [doi: 10.1519/1533-4287(2004)18<551:RAFVOS>2.0.CO;2] [Medline: 15320660] 
28. Cheng KB, Wang C, Chen $\mathrm{H}$, Wu C, Chiu H. The mechanisms that enable arm motion to enhance vertical jump performance-a simulation study. J Biomech 2008;41(9):1847-1854. [doi: 10.1016/j.jbiomech.2008.04.004] [Medline: 18514208]

29. Feltner ME, Fraschetti DJ, Crisp RJ. Upper extremity augmentation of lower extremity kinetics during countermovement vertical jumps. J Sports Sci 1999 Jun;17(6):449-466. [doi: 10.1080/026404199365768] [Medline: 10404494]

30. Harman EA, Rosenstein MT, Frykman PN, Rosenstein RM. The effects of arms and countermovement on vertical jumping. Med Sci Sports Exerc 1990 Dec;22(6):825-833. [doi: 10.1249/00005768-199012000-00015] [Medline: 2287261]

31. Vertical jump test mat. Gill Athletics. URL: https://www.gillporter.com/gill_store/catalog/product/view/id/12661/ [accessed 2021-03-05]

32. Just jump system. Perform Better. URL: https://www.performbetter.com/Just-Jump-System 2 [accessed 2021-03-05]

33. Vertec jump measuring device. Gill Athletics. URL: https://www.gillporter.com/gill_store/vertec-jump-measuring-device. $\underline{\text { html }}$ [accessed 2021-03-05]

34. Electronic vertical jump tester. Gill Athletics. URL: https://www.gillporter.com/gill store/catalog/product/view/id/12673/ [accessed 2021-03-05]

35. Optojump jump testing. Perform Better. URL: https://performbetter.co.uk/product/optojump/ [accessed 2021-03-05]

36. Hawkin dynamics G3 bilateral force plate set. Hawkin Dynamics. URL: https://hawkindynamics.shop/products/ hawkin-dynamics-g3-bilateral-force-plate-set [accessed 2021-03-05]

37. ISO 13485 - medical devices. International Organization for Standardisation. URL: https://www.iso.org/ iso-13485-medical-devices.html [accessed 2021-03-03]

\author{
Abbreviations \\ CMJ: countermovement jump \\ CMJAS: countermovement jump with arm swing \\ FSR: force-sensitive resistor \\ MAE: mean absolute error \\ MRE: mean relative error
}

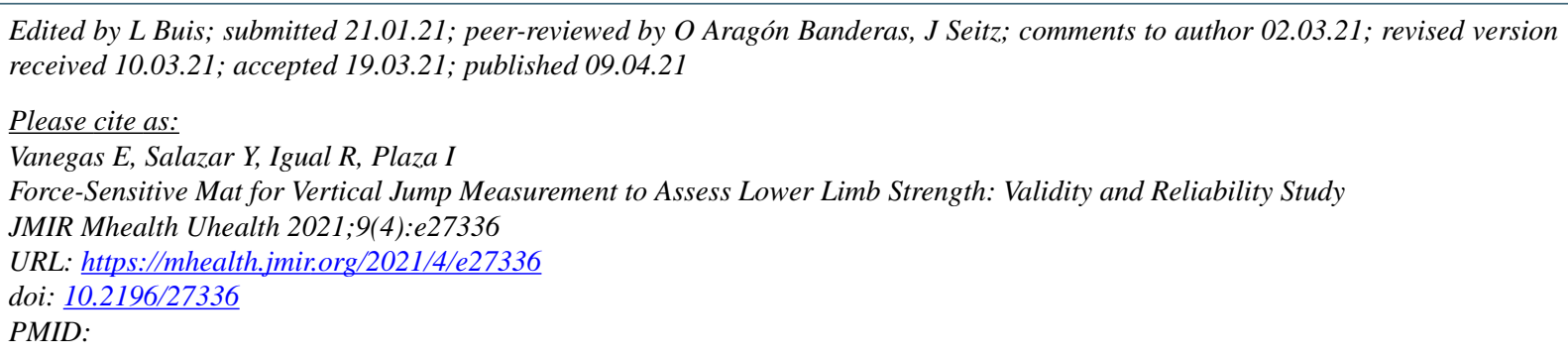

CErik Vanegas, Yolocuauhtli Salazar, Raúl Igual, Inmaculada Plaza. Originally published in JMIR mHealth and uHealth (http://mhealth.jmir.org), 09.04.2021. This is an open-access article distributed under the terms of the Creative Commons Attribution License (https://creativecommons.org/licenses/by/4.0/), which permits unrestricted use, distribution, and reproduction in any medium, provided the original work, first published in JMIR mHealth and uHealth, is properly cited. The complete bibliographic information, a link to the original publication on http://mhealth.jmir.org/, as well as this copyright and license information must be included. 